\title{
Genes controlling essential cell-cycle functions in Drosophila melanogaster
}

\author{
Maurizio Gatti ${ }^{1}$ and Bruce S. Baker ${ }^{2}$ \\ ${ }^{1}$ Dipartimento de Genetica e Biologia Molecolare, Università di Roma 'La Sapienza', 00185 Roma, Italy; ${ }^{2}$ Department of \\ Biological Sciences, Stanford University, Stanford, California 94305 USA
}

On the basis of the hypothesis that mutants in genes controlling essential cell cycle functions in Drosophila should survive up to the larval-pupal transition, 59 such 'late lethals' were screened for those mutants affecting cell division. Examination of mitosis in brain neuroblasts revealed that $\mathbf{3 0}$ of these lethals cause disruptions in mitotic chromosome behavior. These mutants identify genes whose wild-type functions are important for: (1) progression through different steps of interphase, (2) the maintenance of mitotic chromosome integrity, (3) chromosome condensation, (4) spindle formation and/or function, and (5) completion of cytokinesis or completion of chromosome segregation. The presence of mitotic defects in late lethal mutants is correlated tightly with the presence of defective imaginal discs. Thus, the phenotypes of late lethality and poorly developed imaginal discs are together almost diagnostic of mutations in essential cell-cycle functions. The terminal phenotypes exhibited by these Drosophila mitotic mutants are remarkably similar to those observed in mammalian cell-cycle mutants, suggesting that these diverse organisms use a common genetic logic to regulate and integrate the events of the cell cycle.

[Key Words: Cell-cycle mutants; Drosophila; mitosis]

Received November 30, 1988; revised version accepted February 7, 1989.

The exquisitely precise cyclic changes that eukaryotic chromosomes and cells undergo during mitotic and meiotic cell division have been the subject of intense interest for more than 100 years (for review, see Mazia 1961; Pickett-Heaps et al. 1982). Although substantial progress has been made in understanding the individual processes that comprise the chromosome cycle (e.g., replication, repair, chromosome condensation, chromosome movement), we know very little about how these processes are controlled and integrated at a molecular level.

Because all processes that comprise the cell cycle are under genetic control, one approach to understanding these processes is by mutational analysis. This approach has the advantage that the organism tells us which gene products are important for the processes under study. Moreover, a mutational approach should illuminate both structural and regulatory aspects of the cell cycle.

The substantial insights that can be gained from genetic analysis into the functional organization of the cell cycle are evident from elegant studies of Hartwell and his colleagues on cell division cycle (cdc) mutants in Saccharomyces cerevisiae (for review, see Hartwell 1974; Pringle and Hartwell 1981; Moir et al. 1982; Moir and Botstein 1982) and from the more recent studies on Schizosaccharomyces pombe (for review, see Fantes 1984; Nurse 1985). A logic similar to that developed in yeast has been employed to obtain cell-cycle mutations in cultured mammalian cells. However, because of the limitations of mammalian cell cultures for genetic analysis, the events specified by these mutants cannot be ordered easily in temporal and functional sequences (for review, see Simchen 1978; Ling 1981; Oakley 1981; Wissinger and Wang 1983; Marcus et al. 1985).

Thus, it would be advantageous to identify and to analyze a full array of cell-cycle mutations in a multicellular organism that has a well-developed genetics, such as Drosophila melanogaster. The sophisticated genetics of Drosophila should permit the efficient isolation and characterization of mutants. Moreover, there are wellestablished experimental strategies for the subsequent cloning and molecular analysis of genes of interest. Analyzing the cell cycle in an intact multicellular organism has the further advantage that the functions responsible for the temporal and spatial control of mitosis during development would be amenable to analysis. Although a number of mutations with effects on mitotic chromosome behavior have been reported in $D$. melanogaster, with few exceptions these mutations appear to be in genes whose functions are important for the maintenance of chromosome integrity (Baker et al. 1978; Baker and Smith 1979; Gatti 1979; Gatti et al. 1980; Baker et al. 1982; Gatti et al. 1983; Ripoll et al. 1985; Smith et al. 1985; Freeman et al. 1986; Gonzalez et al. 1988; Sunkel et al. 1988). Here we exploit a model for the control of cell division during the life cycle of Drosophila (Baker et al. 1982) to identify mutations in genes that specify a broad array of essential cell-cycle functions.

The fact that Drosophila undergoes complete metamorphosis has important consequences for the organismal phenotypes expected of mutations in essential cell-cycle functions. During Drosophila development there are two essentially independent cell populations that comprise the functional cells of the organism at dif- 
ferent developmental stages: the larval cells that comprise the differentiated structures of the larva and the imaginal cells that are progenitors of the adult. With the exception of a few tissues (e.g., part of the central nervous system), these two cell lineages separate early during embryogenesis. In a Drosophila embryo, the first 10 rounds of mitotic divisions take place very rapidly (cell-cycle time of $\sim 10 \mathrm{~min}$ ) and in the almost complete absence of transcription of the zygotic genome. The cell divisions that produce larval cells are completed by midembryogenesis; subsequent larval growth occurs by an increase in cell size without cell division. In many cases the increase in cell size is accompanied by an increase in chromosome number resulting in polyploidy/polyteny. Thus, it is likely that many, if not all, of the divisions necessary to make the larval cells are accomplished using maternally produced products that are packaged into the egg. Hence, for many cell-cycle genes the maternal contribution may be sufficient to produce a functional complement of larval cells. On the other hand, the imaginal cells divide during the larval period and/or early pupal period and thus almost certainly require the functioning of their own genomes to carry out these divisions. The imaginal cells are completely dispensable for larval growth since organisms lacking these cell populations survive until the larval-pupal transition (Shearn et al. 1971), the stage when the imaginal cells normally differentiate to produce the adult tissues as the larval cells degenerate. Therefore, it appeared possible that the zygotic expression of many cell-cycle functions might not be required for larval viability; however, imaginal cell divisions and the subsequent ability to pupate would require these functions. That is, zygotes homozygous for a cell-cycle mutant, generated by heterozygous (and thus phenotypically wild-type) parents, would be expected to live until about the larval-pupal transition (Baker et al. 1982; Szabad and Bryant 1982). Therefore, this hypothesis predicts that mutant alleles of essential cell-cycle genes should be found among lethals that die at the end of the larval period. Moreover, depending on the type and the severity of the cell-cycle defect they cause, these late lethals should exhibit more or less severely defective imaginal discs.

Here we report the results of examining lethal mutations with lethal phases in the late larval/early pupal period ('late lethals') for their effects on mitotic cell division. These late lethals are an exceedingly rich source of mutants in essential mitotic functions. We cytologically examined cell division in 59 late lethals; of these, 30 cause disruptions in mitotic chromosome behavior. If only late lethals with missing or degenerate imaginal discs are considered, 19 out of the 20 mutants examined affect cell division. Thus, the phenotypes of late lethality and poorly developed imaginal discs are together almost diagnostic of a lesion in an essential cell-cycle function. The cytological characterizations of these mutants have shown that they identify genes whose wildtype functions are important for progression through interphase, the maintenance of chromosome integrity, chromosome condensation, spindle formation and/or function, and the completion of chromosome segregation or cytokinesis.

\section{Results \\ Analysis of nonconditional cell division mutants}

On the basis of our reasoning that lethals causing death during the late larval-early pupal period should be enriched in mutants affecting mitotic cell division, we screened a collection of such lethals (late lethals) for mutants affecting the cell cycle. In attempting to identify mutants affecting mitosis in a multicellular eukaryote, several problems need to be faced that are not encountered in systems where the organism being used is single celled in fact (e.g., yeast) or in practice (e.g., mammalian tissue culture cells). In the latter systems the identification and characterization of cell division cycle mutants has depended heavily on (1) the availability of essentially homogeneous cell populations, (2) the ability to follow the descendents of single cells, and (3) the recovery of conditional (e.g., temperature-sensitive) mutants. These features allow a cell-cycle mutant to be identified when all cells in a mutant population exhibit a common terminal phenotype under the restrictive condition. Here none of these simplifying features is available. Instead, we are faced with the identification and analysis of nonconditional mutants in a multicellular organism without determined cell lineages.

There are a number of important ways that the biology of this situation significantly complicates both the identification and analysis of mutants affecting cell division. First, the fact that the mothers of the homozygous lethal individuals being examined carry a wildtype copy of the gene being studied means that there is maternally produced wild-type gene product in the mutant individuals. Thus, an individual homozygous for a cell-cycle mutant may display a normal cell-cycle phenotype for some portion of its development because of the persistence of the maternally contributed wild-type gene product. The extent of time across which this occurs may vary substantially between tissues because proliferation occurs at different times in different tissues. The persistence of maternal products can also lead to heterogeneity within tissues because growth of different cell lineages may be by mechanisms that dilute maternal products to differing extents (e.g., stem cell divisions versus exponential growth). In part to minimize these problems, we decided to look for effects of these mutants on the cell cycle in a single tissue late in development - the ganglia of mature third-instar larvae.

By looking at homozygous mutants late in development, we should be observing cells in which the mutant is exhibiting a terminal phenotype because of the absence of an essential function for a number of cell cycles. Ideally, in this situation all cells should display comparable phenotypes. In practice this expectation may not be met because a mutant in an essential cell-cycle function may be completely tight in terms of its organismal lethal phenotype, but leaky in terms of its cellular phe- 
notype. Thus, some cells may exhibit a unique cellular phenotype, whereas others will appear normal. The chance of identifying such leaky mutants is a function of both the degree of leakiness and the distinctiveness of the terminal phenotype. However, once identified, such leaky mutants can be particularly informative in that all cells are not arrested with the same terminal phenotype. Thus, it is possible to monitor the effects of such mutants on the normality of a number of different steps (landmarks) in the chromosome (or cell) cycle. This can aid both in delimiting when the wild-type function of a gene becomes required for the normal progression of the cell cycle and in distinguishing between the wild-type functions of genes in which mutants produce similar terminal phenotypes.

\section{Initial screen for mutants affecting the cell cycle}

With these considerations in mind, mitotic cell division was examined in individuals homozygous for $15 \mathrm{X}$ linked and 42 third-chrosomome late lethals that had been isolated previously (Shearn et al. 1971; Stewart et al. 1972; Shearn and Garen 1974; Kiss et al. 1976).

Initially, we screened aceto orcein squash preparations of larval ganglia treated for $1.5 \mathrm{hr}$ with colchicine. For each mutant, at least five homozygous mutant ganglia and five control ganglia of similar sizes were examined. To minimize treatment artifacts, mutant and control ganglia were dissected from larvae of the same culture, treated together, and then squashed at well-separated locations on the same set of slides. For X-linked lethals, control ganglia were Binsn $/ Y$ males or Binsn/mutant heterozygous females; for third chromosome lethals, controls were $T M 6 b /$ mutant heterozygotes. Control and mutant ganglia were distinguished on the basis of chromosome morphology: the multiply inverted Binsn and $T M 6 b$ balancer chromosomes are distinct morphologically from normal sequence chromosomes (Figs. la and 3a). A medium-sized ganglion from a third-instar control larva usually exhibits $200-400$ metaphases with wellspread chromosomes (Figs. 1a, 3a, and 4b). Thus, a comparison of control and mutant ganglia enabled us to detect aneuploidy and polyploidy as well as to monitor both chromosome integrity and chromosome condensation. In addition, these observations provided a rough estimate of the mitotic index in mutant ganglia. The results of these analyses are summarized in Table 1. There also we include the results of similar examinations of mus-105 and mus-109ls, two late lethals studied previously (Baker et al. 1982).

These data show that our general expectation - that mutants affecting mitotic cell division would be represented among late lethals -is amply met: 29 of the 59 mutants examined had effects on one or more of the parameters of chromosome behavior that were measured. Moreover, these data reveal a striking correlation between the effects of these lethals on imaginal discs and whether they show effects on mitotic cell division in ganglia. These late lethal mutants have been divided into four classes on the basis of the appearance of ima-

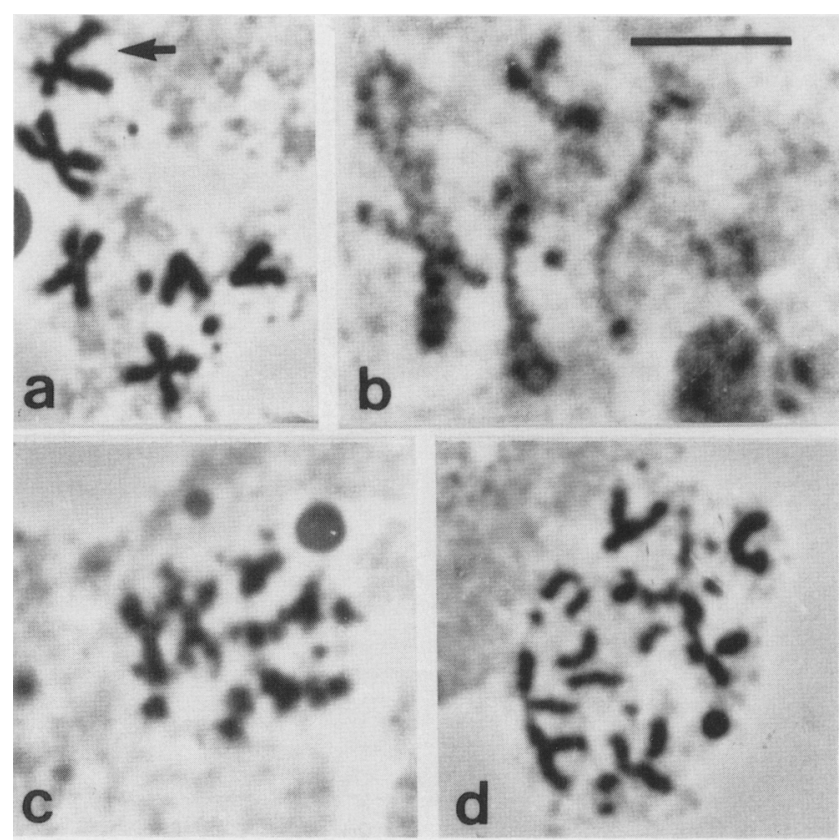

Figure 1. Colchicine-treated metaphases observed in mutants with few cells in division. (a) A normal metaphase in I(3)e20/TM6b; note the submetacentric TM6b balancer (arrow). (b) Irregularly condensed metaphase in 1(3)e20. (c) Irregularly condensed and fragmented metaphase in 1(3)1902. (d) Extensive chromosome breakage in mus-109Is. Bar, $5 \mu \mathrm{m}$.

ginal discs in homozygous mutant larvae: (1) those in which imaginal discs are missing or degenerate, (2) those in which some discs are missing and others are normal, (3) those with small imaginal discs, and (4) those in which imaginal discs appear normal. Of the late lethals in the missing and/or degenerate imaginal disc category, 19 out of 20 affect at least one important aspect of mitotic cell division. However, of the 21 late lethals exhibiting either small imaginal discs (19 mutants) or heterogeneous discs (2 mutants), only 10 exhibit defects in cell division, whereas only one of the 18 mutants that had normal discs showed a discernible effect on mitosis. Thus, there is a strong correlation between the phenotypes of defective mitotic cell division in the larval ganglia and poorly developed imaginal discs. This suggests that these phenotypes are related causally and that the presence of small/degenerate discs is, in most cases, the result of the impairment of an essential cell-cycle function.

These initial observations also allowed us to divide these mutants into five classes on the basis of the occurrence and kinds of mitotic effects that are observed. Four major categories of mutants that affect the cell cycle are distinguishable: (1) mutants with few or no dividing cells, (2) mutants affecting chromosome condensation, (3) mutants arresting the cell cycle at metaphase, and (4) mutants producing division failures that result in highly polyploid cells. The findings on individual mutants in these four categories are described below, together with the results of additional experiments that help elucidate 
Table 1. Properties of lethal mutants affecting mitotic cell division in larval ganglia

\begin{tabular}{|c|c|c|c|c|c|c|c|}
\hline \multirow[b]{2}{*}{ Mutant } & \multirow[b]{2}{*}{ Reference $^{\mathbf{a}}$} & \multirow[b]{2}{*}{$\mathrm{Map}^{\mathrm{b}}$} & \multirow{2}{*}{$\begin{array}{l}\text { Disc } \\
\text { size }^{c}\end{array}$} & \multirow{2}{*}{$\begin{array}{l}\text { Mitotic } \\
\text { index }^{d}\end{array}$} & \multicolumn{3}{|c|}{ Mitotic defect ${ }^{\mathrm{e}}$} \\
\hline & & & & & condensation & breakage & polyploidy \\
\hline 1(1) discless $-1^{\mathrm{f}}$ & 1,2 & $1-35$ & $\mathrm{~m} / \mathrm{d}$ & $\mathrm{nm}$ & & & \\
\hline $1(1) C 154^{f}$ & 3 & $1-35$ & $\mathrm{~m} / \mathrm{d}$ & $\mathrm{nm}$ & & & \\
\hline $1(1) d$ deg-1g & 4,5 & $1-13.7$ & $\mathrm{~m} / \mathrm{d}$ & 1 & - & +++ & - \\
\hline 1(1)d deg-3 & 4 & $1-27.5$ & $\mathrm{~m} / \mathrm{d}$ & $\mathrm{h}$ & +++ & ++ & +++ \\
\hline $1(1) d$ deg-4 & 4 & $1-52$ & $\mathrm{~m} / \mathrm{d}$ & $\mathrm{h}$ & - & - & +++ \\
\hline 1(1)d deg-9 & 1 & $1-29.7$ & $\mathrm{~m} / \mathrm{d}$ & 1 & ++ & ++ & + \\
\hline $1(1) d$ deg-10 & 1 & $1-46.7$ & $\mathrm{~m} / \mathrm{d}$ & $\mathrm{h}$ & +++ & ++ & +++ \\
\hline $1(1) d \operatorname{deg}-11$ & 1 & $1-27.2$ & $\mathrm{~m} / \mathrm{d}$ & $\mathrm{c}$ & - & + & +++ \\
\hline $1(1) d$ deg-12 & 1,2 & $1-65.8$ & $\mathrm{~m} / \mathrm{d}$ & 1 & ++ & - & + \\
\hline mus-109ls g & 5,6 & $1-29$ & $\mathrm{~m} / \mathrm{d}$ & $\mathrm{vl}$ & - & +++ & - \\
\hline $1(3) 7 m-147$ & 7 & $3-68.7$ & $\mathrm{~m} / \mathrm{d}$ & $\mathrm{c}$ & - & - & - \\
\hline $1(3) 8 m-12$ & 7 & $3-46.4$ & $\mathrm{~m} / \mathrm{d}$ & 1 & + & - & - \\
\hline $1(3) 11 m-254$ & 7 & $3-49.3$ & $\mathrm{~m} / \mathrm{d}$ & 1 & + & - & - \\
\hline $1(3) 12 m-137$ & 7 & $3-130.2$ & $\mathrm{~m} / \mathrm{d}$ & 1 & + & - & - \\
\hline $1(3) 13 m-230$ & 7 & $3-5.5$ & $\mathrm{~m} / \mathrm{d}$ & & ++ & ++ & - \\
\hline $1(3) 15 m-25$ & 7 & $3-58.2$ & $\mathrm{~m} / \mathrm{d}$ & 1 & ++ & + & - \\
\hline $1(3) e 20$ & 7 & $3-25.9$ & $\mathrm{~m} / \mathrm{d}$ & $\mathrm{vl}$ & ++ & +++ & - \\
\hline $1(3) K 43$ & 7 & $3-51.1$ & $\mathrm{~m} / \mathrm{d}$ & $\mathrm{vl}$ & ++ & +++ & - \\
\hline $1(3) 2004$ & 7 & $3-50.3$ & $\mathrm{~m} / \mathrm{d}$ & $\mathrm{c}$ & ++ & + & ++ \\
\hline $1(3) I I b 1$ & 7 & $3-70.4$ & $\mathrm{~m} / \mathrm{d}$ & 1 & + & - & - \\
\hline 1(1)het2 & 1 & $1-57$ & $\mathrm{~m} / \mathrm{n}$ & 1 & ++ & ++ & + \\
\hline $1(3) / 16$ & 7 & 3-15.6 & $\mathrm{m} / \mathrm{s}$ & $\mathrm{c}$ & - & - & - \\
\hline $1(1) C 248$ & 3 & $1-35$ & $\mathrm{~s}$ & $\mathrm{c}$ & - & - & - \\
\hline l(1)d sml-8 & 1 & $1-19.5$ & $\mathrm{~s}$ & $\mathrm{c}$ & - & - & - \\
\hline 1(1)d sml-9 & 1 & $1-57$ & s & $\mathrm{c}$ & - & - & - \\
\hline $1(3) 7 m-62$ & 7 & $3-36.8$ & $\mathrm{~s}$ & c & - & ++ & +++ \\
\hline $1(3) 7 m-68$ & 7 & $3-51.8$ & $\mathrm{~s}$ & 1 & - & - & - \\
\hline $1(3) 13 m-237$ & 7 & $3-2.4$ & $\mathrm{~s}$ & $\mathrm{c}$ & - & - & - \\
\hline $1(3) 1902$ & 7 & $3-30.9$ & $\mathrm{~s}$ & $\mathrm{vl}$ & + & +++ & - \\
\hline 1(3)C21 & 7 & $3-62.5$ & $\mathrm{~s}$ & $\mathrm{c}$ & - & - & - \\
\hline $1(3) I X-11$ & 7 & $3-81.7$ & s & $\mathrm{vl}$ & + & - & - \\
\hline $1(3) X I I-10$ & 7 & $3-76.6$ & $\mathrm{~s}$ & 1 & + & ++ & + \\
\hline $1(3) 517$ & 7 & $3-35.7$ & $s$ & c & - & - & - \\
\hline $1(3) 2306$ & 7 & $3-74.0$ & $\mathrm{~s}$ & c & - & - & - \\
\hline $1(3) 2612$ & 7 & $3-48.7$ & $\mathrm{~s}$ & 1 & +++ & - & + \\
\hline $1(3) I X-14$ & 7 & $3-49.0$ & $\mathrm{~s}$ & 1 & ++ & + & - \\
\hline $1(3) 7 m-75$ & 7 & $3-53.8$ & $\mathrm{~s}$ & 1 & ++ & + & - \\
\hline
\end{tabular}

(Continued on next page.)

the functions of these genes. In addition, there are 28 mutants that do not have discernible effects on the structure or number of mitotic chromosomes and do not substantially affect the mitotic index. One mutant included in this category, $1(3) 7 m-68$, exhibited somewhat fewer dividing cells than its control, but these metaphases appeared completely normal.

\section{Mutants with few or no cells in division}

In eight mutants representing seven genes there are few or no dividing cells in larval brains. These mutants, which have mitotic indices designated " $\mathrm{nm}$ " or " $\mathrm{vl}$ " in Table 1, have been grouped together here solely on the basis of their low mitotic indices. To obtain more accurate estimates of the degree to which these lethals reduce the frequencies of dividing cells, at least 10 additional mutant ganglia and 10 additional control ganglia were examined for each mutant. In all cases but two, [1(1)discless-1 and its allele 1(1)C154 in which no divi- sion figures were observed], the mitotic index is reduced about two orders of magnitude relative to controls. Thus, these mutants identify a set of genes whose wildtype functions are necessary for the occurrence of mitosis; cells accumulate in interphase in the absence of these functions. It is not known whether these mutants lead to arrests at a particular point in interphase. However, an examination of the few mitotic figures found in these mutants revealed characteristic abnormalities suggesting that these genes have a variety of functions.

In four of these mutants there are substantial effects on chromosomal integrity seen in the few dividing cells that were observed. Thus, in 1(3)e20 and 1(3) K43, chromosomes are irregularly condensed and extensive chromosome fragmentation is observed frequently (Fig. 1). In 1(3)1902, chromosome fragmentation is also frequent but chromosome condensation is only slightly abnormal (Fig. 1). An extremely low mitotic index also was found in mus-109's (Baker et al. 1982). We screened 10 mus-109's mutant brains to determine the mitotic phe- 
Table 1. Continued

\begin{tabular}{|c|c|c|c|c|c|c|c|}
\hline \multirow[b]{2}{*}{ Mutant } & \multirow[b]{2}{*}{ Reference $^{a}$} & \multirow[b]{2}{*}{$\mathrm{Map}^{\mathrm{b}}$} & \multirow{2}{*}{$\begin{array}{l}\text { Disc } \\
\text { size }^{c}\end{array}$} & \multirow{2}{*}{$\begin{array}{l}\text { Mitotic } \\
\text { index }^{\mathrm{d}}\end{array}$} & \multicolumn{3}{|c|}{ Mitotic defect ${ }^{e}$} \\
\hline & & & & & condensation & breakage & polyploidy \\
\hline $1(3) 13 m-281$ & 7 & $3-26.6$ & $\mathrm{~s}$ & $\mathrm{vl}$ & - & - & +++ \\
\hline $1(3) 15 m-15$ & 7 & $3-37.8$ & $\mathrm{~s}$ & $\mathrm{c}$ & - & - & - \\
\hline $1(3) 15 m-33$ & 7 & $3-24.0$ & $\mathrm{~s}$ & c & - & - & - \\
\hline $1(3) \mathrm{g} 60 \mathrm{~A}$ & 7 & $3-81.4$ & $\mathrm{~s}$ & 1 & ++ & + & - \\
\hline $1(3) \mathrm{m} 45$ & 7 & $3-49.5$ & ad & 1 & + & - & - \\
\hline $1(3) V-13$ & 7 & $3-$ & ad & c & - & - & - \\
\hline $1(3) 2614$ & 7 & 3- & ad & c & - & - & - \\
\hline $1(3) g 21$ & 7 & $3-52.1$ & ad & $\mathrm{c}$ & - & - & - \\
\hline $1(3) g 49$ & 7 & $3-39.4$ & ad & c & - & - & - \\
\hline $1(3) \mathrm{K} 60$ & 7 & $3-$ & ad & c & - & - & - \\
\hline $1(3) \mathrm{m} 47$ & 7 & $3-4.3$ & ad & c & - & - & - \\
\hline $1(3) C 43-3$ & 7 & $3-49.0$ & ad & c & - & - & - \\
\hline l(1)d norm-12 & 1 & $1-02$ & $\mathrm{n}$ & c & - & - & - \\
\hline 1(1)d norm-15 & 1 & $1-9.5$ & $\mathbf{n}$ & c & - & - & - \\
\hline 1(1)d norm-25 & 1 & $1-22$ & $\mathrm{n}$ & c & - & - & - \\
\hline $1(3) C 21 L$ & 7 & $3-48.7$ & $\mathrm{n}$ & c & - & - & - \\
\hline $1(3) g 30 R$ & 7 & $3-79.8$ & $\mathrm{n}$ & c & - & - & - \\
\hline$l(3) g 131$ & 7 & $3-$ & n & c & - & - & - \\
\hline $1(3) / 151$ & 7 & $3-25.5$ & $\mathrm{n}$ & $\mathrm{c}$ & - & - & - \\
\hline $1(3) L 36$ & 7 & $3-62.4$ & $\mathrm{n}$ & $\mathrm{c}$ & - & - & - \\
\hline $1(3) \mathrm{m} 27$ & 7 & $3-23.3$ & $\mathrm{n}$ & c & - & - & - \\
\hline $1(3) 1803 R 16$ & 7 & $3-59.3$ & $\mathrm{n}$ & c & - & - & - \\
\hline
\end{tabular}

a (1) Kiss et al. 1976; (2) Kiss et al. 1978; (3) obtained from G. Lefevre; (4) Stewart et al. 1972; (5) Baker et al. 1982; (6) obtained from A. Schalet; (7) Shearn et al. 1971; Shearn and Garen 1974; Lindsley and Zimm 1986.

b Chromosome and meiotic map position of mutants.

c $(\mathrm{m} / \mathrm{d})$ Missing/degenerate: extreme reduction of disc tissue; (s) small: reduction in size of disc tissue; (ad) abnormal differentiation: morphologically normal discs that differentiate abnormally; (n) normal: normal discs; $(\mathrm{m} / \mathrm{n}) \mathrm{missing} / \mathrm{normal}$ : some discs are extremely reduced and others are normal; $(\mathrm{m} / \mathrm{s}) \mathrm{missing} / \mathrm{small}$ : some discs are extremely reduced and others are small.

${ }^{d}(\mathrm{~nm})$ No mitoses: complete absence of dividing cells. (vl) very low: (MI) Mitotic Index between 1 and $10 \%$ of the control value; (l) low, MI between 10 and $50 \%$ of control; (c) control, MI from 0.5 to 1.5 times control; (h) high, MI $>1.5$ times control. (Blank) MI not determined.

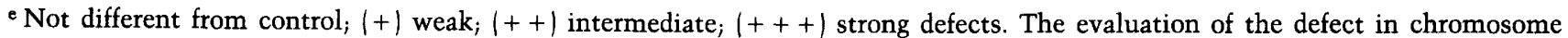
condensation is based on both the degree of departure from normality and the frequency of affected cells. For chromosome breakage and polyploidy, $(+)$ between 2 and $10 \%$ of metaphases exhibit the trait; $1++\mid$ and $1+++\mid$ between 10 and $20 \%$ and $>20 \%$ of metaphases exhibit the trait, respectively.

${ }^{f} 1(1) d$ discless-1 and 1(1)C154 are allelic.

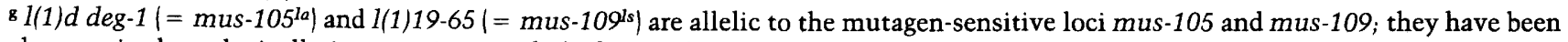
characterized cytologically in a previous study (Baker et al. 1982).

notype. These brains yielded only four dividing cells, each of which exhibited extensive chromosome breakage but no clear defects in chromosome condensation (one of these cells is shown in Fig. 1).

The mutant 1(3)IX-11 exhibits a low mitotic index comparable to those of the above mutants but does not affect chromosome integrity. The few metaphases observed in 1(3)IX-11 have quasi-normal chromosomes with somewhat thin and elongated chromatids. This effect on chromosome condensation is sufficiently weak that we do not feel it should be used to make any inference about the cell-cycle function specified by $1(3) I X-11$.

The few mitotic figures observed in 1(3)13m-281 are always endoreduplicated (Fig. 2). (An endoreduplicated cell is a polyploid cell in which sister chromosomes are paired.) Different cells exhibited different degrees of endoreduplication, with bundles of 4,8 , or 16 sister chromosomes. Because endoreduplication is believed to result from successive rounds of DNA replica-

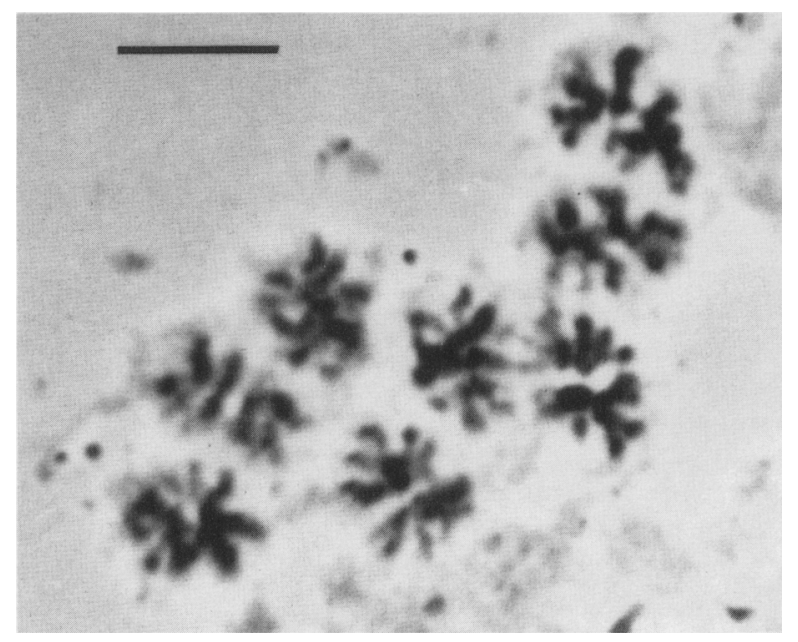

Figure 2. Endoreduplicated metaphase in $1(3) 13 m-281$. Bar, 5 $\mu \mathrm{m}$. 
tion with no mitotic division occurring between them (Schwarzaker and Schnedl 1965; Nagl 1978), the wildtype allele of $1(3) 13 m-281$ may specify a cell cycle function required for either the progression through $\mathrm{G}_{2}$ or the onset of mitosis.

Finally, 1(1)discless-1 and its allele 1(1)C154 do not exhibit any mitotic figures; only interphase nuclei are seen in their ganglia.

\section{Mutants affecting chromosome condensation}

Fifteen of these mutants have substantial effects on chromosome condensation. Two other genes that specify functions necessary for normal chromosome condensation have been previously reported in Drosophila (Gatti et al. 1983; Smith et al. 1985). The genes identified here have wild-type functions that are needed for the normal condensation of both euchromatin and heterochromatin and are, thus, like one of the previously identified genes, mit (1)4. However, the function of the other previously identified gene, mus-101, is distinct from the functions of the genes reported here, in that its wild-type function is necessary for the condensation of heterochromatic but not euchromatic portions of the genome (Gatti et al. 1983). The phenotypes of the mutants affecting condensation that we report here let them be subdivided into several categories on the basis of similarities in their phenotypes. These are as follows.

Two mutants, 1(1)d deg-12 and 1(3)2612, exhibit swollen and unevenly condensed chromosomes. Also, coiling of chromatids can be seen frequently. In addition, in both mutants $\sim 10 \%$ of metaphases are extremely hyperploid and/or tetraploid (Fig. 3).

Four other mutants, $1(3) I X-14,1(3) g 60 A, 1(3) 7 m-75$, and $1(3) 15 m-25$, also exhibit swollen and unevenly condensed chromatids. However, in these mutants defective chromosome condensation is not accompanied by polyploidy but rather by the presence of low levels of chromosome breakage.

Two mutants, $1(1)$ het-2 and $1(3) 2004$, show a combination of the phenotypes displayed by the two previous categories of mutants. In these two mutants chromatids are slightly swollen and irregularly condensed and both chromosome breakage and polyploid cells are observed (Fig. 3).

Tetraploid cells and chromosome breakage also are observed in 1(1)d deg-9 and l(3)XII-10, but these mutants differ from each other and from those described above in their effects on chromosome condensation. In 1(1)d deg-9 chromosomes are swollen, fuzzy, and in some cases, unevenly condensed; sister chromatids often are closely apposed; heterochromatic regions are slightly elongated and understained and the Y chromosome consistently exhibits a long undercondensed region (Fig. 3). This phenotype is reminiscent of that of mit(1)4, a temperature-sensitive lethal affecting chromosome condensation (Smith et al. 1985). The map positions of mit(1)4 and 1(1)d deg-9 are compatible with the possibility that these mutants are allelic. In 1(3)XII-10 chromosome condensation is only slightly affected; chromatids are

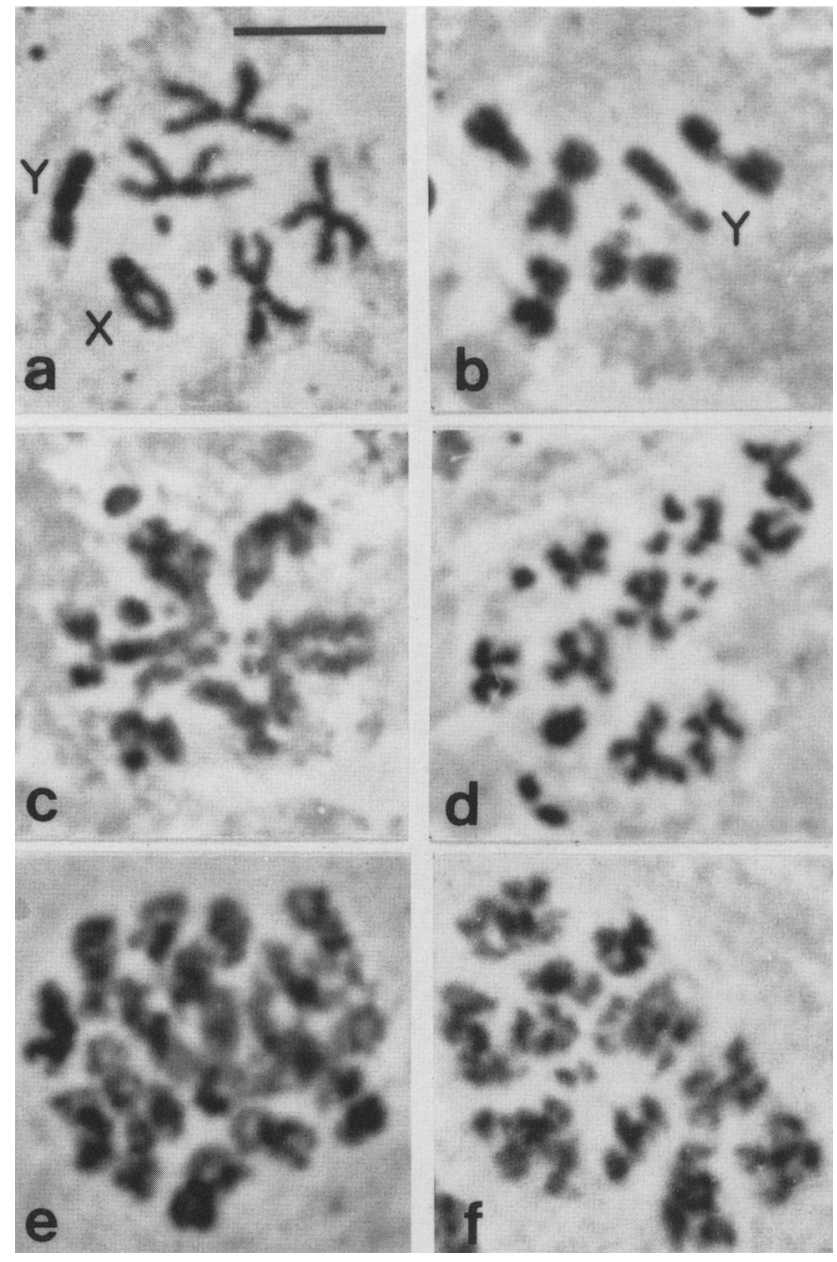

Figure 3. Colchicine-treated metaphases in mutants affecting chromosome condensation. (a) Control Binsn/Y metaphase. $(b)$ Irregularly condensed metaphase in $1(1) d$ deg-9. Note the long unstained gap in the Y chromosome. $(c, d)$ Irregularly condensed metaphases in $1(1) d$ het-2; $d$ is clearly hyperploid and exhibits extensive chromosome breakage. $(e, f)$ Irregularly condensed polyploid/hyperploid metaphases in 1(3)2612. Bar, $5 \mu \mathrm{m}$.

often unevenly condensed and tend to be thinner than normal.

Finally, there are five mutants, $1(3) 8 \mathrm{~m}-12,1(3) 11 \mathrm{~m}$ $254,1(3) 12 m-137,1(3) I I b 1$, and $1(3) m 45$, that exhibit mild defects in chromosome condensation. In these mutants, in addition to normal metaphases, there are metaphases in which chromosomes are slightly swollen and fuzzy and, in some cases, unevenly condensed. Neither chromosome breakage nor polyploid cells have been observed in these mutants.

The range of effects produced by these 15 mutants indicates that they are defective in a variety of functions; how directly the wild-type functions of these genes are concerned with chromatin condensation is considered in the Discussion section. 


\section{Mutants arresting the cell cycle at metaphase}

The initial screens of the colchicine-treated ganglia from six mutants [1(1)d deg-3, 1(1)d deg-4, 1(1)d deg-10, 1(1)d deg-11, $1(3) 7 m-62$, and $1(3) 13 m-230)$ revealed higher than normal mitotic indices and/or frequent polyploid cells. These observations suggested that mitosis itself might be defective in these mutants. To characterize the mitotic phenotypes of these mutants, squash preparations were made omitting the colchicine and hypotonic pretreatments. Colchicine prevents polymerization of spindle microtubules and thus causes a metaphase block that leads to contracted chromosomes not organized on a metaphase plate. Hypotonic treatment causes nuclear swelling and a separation of sister chromatids, and drastically reduces the frequency of anaphases (Brinkley et al. 1980). The combination of these treatments leads to the accumulation of metaphases with contracted and scattered chromosomes with well-separated sister chromatids, which remain attached at the centromere (see Fig. 4). In the preparations from which colchicine and hypotonic treatments are omitted, cells are not blocked in metaphase and all phases of mitosis can be analyzed. By comparing nontreated mutant and control brains squashed on the same slide, we were able to subdivide these mutants into those that arrest at metaphase (discussed in this section) and those that have normal mitotic indices and exhibit normal anaphases but produce polyploid cells (discussed below).

The mutants that result in the arrest of cells at metaphase can be placed into three subclasses with respect to the terminal phenotypes that they exhibit.

The first class contains one mutant, $1(1) \mathrm{d}$ deg-4. In this mutant the mitotic index in noncolchicine-treated cells is three times higher than that in the control, suggesting that cells are accumulating at metaphase (Table 2). Most metaphase figures observed in noncolchicinetreated 1(1)d deg-4 cells have contracted and scattered chromosomes resembling colchicine-induced metaphases (c-metaphases) (cf. Fig. 5 with Fig. 4). Thus, these chromosomes are overcondensed compared to controls. In 1(1)d deg-4, sister chromatids are well separated but usually remain attached at the centromere. Anaphases in 1(1)d deg-4 are very rare (Table 2 ) and the few detected

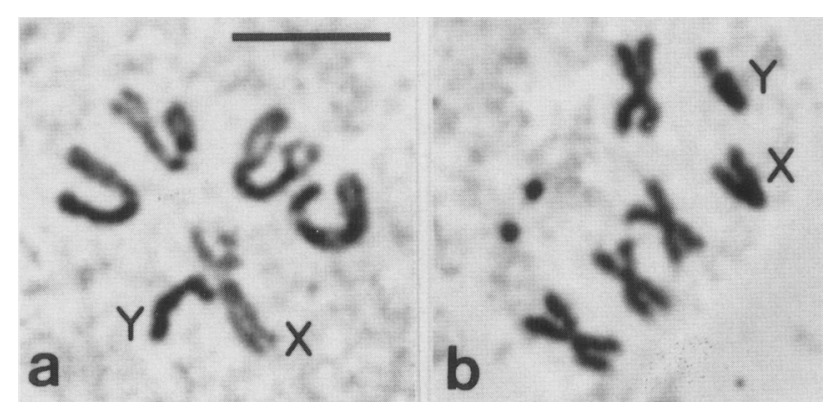

Figure 4. Control male metaphases. (a) Cell from a brain without colchicine and hypotonic pretreatments. $(b)$ Cell from a brain incubated for $1.5 \mathrm{hr}$ with colchicine and then treated with hypotonic solution. Bar, $5 \mu \mathrm{m}$. are abnormal: The poles are broad and chromosomes are frequently widely scattered (Fig. 5 ). In addition, $\sim 25 \%$ of metaphase figures are tetraploid or hyperploid. Cells with higher degrees of ploidy $(8 n, 16 n)$ are relatively rare. The presence of polyploid cells suggests that cells arrested in metaphase sometimes revert to interphase and undergo additional chromosome doubling. These observations suggest strongly that $1(1) d$ deg-4 specifies a function necessary for spindle formation and/or function.

The second class of metaphase arrest mutants, $1(1) d$ deg-3 and 1(1)d deg-10, also show a threefold increase in the frequency of metaphases and exhibit no anaphases (Table 2). Metaphase figures in noncolchicine-treated cells from these mutants have extremely condensed chromosomes that are widely scattered (Fig. 6). Thus, in these mutants, as in 1(1)d deg-4, there is not a wellformed metaphase plate. The degree of metaphase chromosome condensation observed in these two mutants is even greater than that produced by prolonged colchicine treatments of wild type. In these two mutants sister chromatids almost always are separated and not connected at the centromere (Fig. 6). In addition, many cells [ $\sim 30 \%$ in $1(1) \mathrm{d} \operatorname{deg}-10$ and $20 \%$ in $1(1) \mathrm{d} \mathrm{deg}-3]$ exhibit low levels of polyploidy $(4 n, 8 n)$ and/or hyperploidy, indicating that in these mutants, as in $1(1) d$ deg-4, arrested cells may revert to interphase and double their chromosome complement. Finally, in both mutants chromosome fragmentation is common and usually separates the chromosome into two approximately equal-sized pieces, suggesting that breakage occurs at or near the centromere. Although these observations clearly show that, in both these mutants, cells arrest at metaphase, they do not suggest any simple hypothesis as to the mechanism(s) underlying this arrest. The fact that most mitotic figures observed in $1(1) d$ deg-3 and $l(1) d$ deg-10 are much more condensed than the mitotic figures seen in 1(1)d deg-4 indicates that there is an important difference between the metaphase blocks in these mutants.

Another mutant that exhibits a mitotic phenotype similar to that seen in 1(1)d deg-3 and l(1)d deg-10 is $1(3) 13 m-230$. In this mutant we also observed both a high frequency of metaphase figures with scattered, overcondensed chromosomes having separated sister chromatids, as well as frequent chromosome breakage at or near the centromere (Fig. 6). However, in $1(3) 13 m-230$ there were few or no polyploid cells. Unfortunately, $1(3) 13 m-230$ could not be fully characterized because it was lost (effectively simultaneously in three different laboratories!) before completion of cytological analysis.

\section{Mutants producing highly polyploid cells}

The two mutants in this class, $1(1) \mathrm{d} \mathrm{deg-11}$ and $1(3) 7 \mathrm{~m}$ 62 , also lead to the production of polyploid cells. However, the phenotypes of these mutants differ from those of the mutants described in the preceding section in a number of important parameters. Both 1(1) d deg-11 and $1(3) 7 m-62$ have mitotic indices comparable to control, do not exhibit metaphase plates with scattered and highly condensed chromosomes (c-metaphases) and 
Table 2. Mitotic indices and frequencies of anaphases in mutants producing polyploid cells

\begin{tabular}{|c|c|c|c|c|c|c|c|c|c|c|}
\hline \multirow[b]{2}{*}{ Mutant } & \multirow[b]{2}{*}{$\begin{array}{l}\text { Number } \\
\text { of brains }\end{array}$} & \multirow{2}{*}{\multicolumn{2}{|c|}{$\begin{array}{c}\text { Number } \\
\text { of metaphases }\end{array}$}} & \multicolumn{3}{|c|}{$\begin{array}{c}\text { Number } \\
\text { of anaphases }\end{array}$} & \multirow[b]{2}{*}{$\begin{array}{l}\text { Cells } \\
\text { scored }\end{array}$} & \multirow{2}{*}{$\begin{array}{l}\text { Polyploid } \\
\text { figures } \\
(\%)\end{array}$} & \multirow[b]{2}{*}{$\begin{array}{l}\text { Anaphases } \\
(\%)\end{array}$} & \multirow{2}{*}{$\begin{array}{l}\text { Divisions } \\
\text { per optic } \\
\text { field }^{\mathrm{a}}\end{array}$} \\
\hline & & & $\frac{\text { aphases }}{\text { polyploid }}$ & diploid & polyploid & $\begin{array}{l}\text { multipolar } \\
\text { polyploid }\end{array}$ & & & & \\
\hline \multirow{2}{*}{$\begin{array}{l}\text { A. } 1(1) d \text { deg-4 } \\
\text { Binsn }\end{array}$} & 19 & 1212 & 372 & 8 & 1 & 0 & 1593 & 23.4 & 0.6 & 1.63 \\
\hline & 15 & 416 & 0 & 70 & 0 & 0 & 486 & 0 & 14.4 & 0.48 \\
\hline \multirow{2}{*}{$\begin{array}{l}\text { B. } 1(1) d \text { deg-10 } \\
\text { Binsn }\end{array}$} & 18 & 849 & 373 & 0 & 0 & 0 & 1222 & 30.5 & 0 & 1.52 \\
\hline & 19 & 551 & 0 & 90 & 0 & 0 & 641 & 0 & 14.0 & 0.54 \\
\hline \multirow{2}{*}{$\begin{array}{l}\text { C. } 1(1) d \text { deg-3 } \\
\text { Binsn }\end{array}$} & 18 & 519 & 135 & 0 & 0 & 0 & 654 & 20.6 & 0 & 1.40 \\
\hline & 19 & 638 & 0 & 101 & 0 & 0 & 739 & 0 & 13.7 & 0.45 \\
\hline \multirow{2}{*}{$\begin{array}{l}\text { D. } 1(1) d \text { deg-11 } \\
\text { Binsn }\end{array}$} & 14 & 227 & 170 & 35 & 26 & 6 & 464 & 43.5 & 14.4 & 0.60 \\
\hline & 15 & 426 & 0 & 76 & 0 & 0 & 502 & 0 & 15.1 & 0.52 \\
\hline \multirow{2}{*}{ E. $\begin{array}{ll}1(3) 7 m-62 \\
1(3) 7 m-62 / T M 6 b\end{array}$} & 16 & 263 & 583 & 41 & 58 & 11 & 956 & 68.2 & 11.5 & 0.64 \\
\hline & 21 & 845 & 0 & 122 & 0 & 0 & 967 & 0 & 12.6 & 0.63 \\
\hline
\end{tabular}

a In each brain mitotic figures were scored in every fourth optic field; see Methods.

The nonmutant immediately below each mutant genotype is its parallel sib control.

have normal frequencies of anaphases (Table 2; Fig. 7), indicating that these two mutants do not produce metaphase arrests.

In these two mutants both the frequency of polyploid nuclei and the degree of polyploidy seen in a cell can be quite high. Thus, in the $1(3) 7 m-62$ ganglia that we scored, $\sim 70 \%$ of metaphases are polyploid/hyperploid, whereas in 1(1)d deg-11, $\sim 40 \%$ of cells are polyploid/hyperploid. In addition, in both mutants, cells containing 500-1000 chromosomes are found (Fig. 8). However, these observations, with respect to the frequencies and degree of polyploidy attainable in these mutants, must be viewed from the perspective that larvae homozygous for either of these mutants do not die immediately on reaching the end of the larval period, but, instead, remain as third-instar larvae for several days longer than their wild-type sibs. Thus, in these older larvae, some of which were certainly included among the larvae from which we made our chromosome preparations, cells have had substantially more time to become polyploid than would cells in wild type or in mutants that died more immediately on reaching the larval-pupal transition. Nevertheless, to generate the high levels of polyploidy seen in some cells in these mutants, six to seven complete failures of chromosome segregation within a cell lineage are needed. However, as discussed below, this does not necessarily mean that the cell-cycle defect is manifest in each cell division in these lineages.

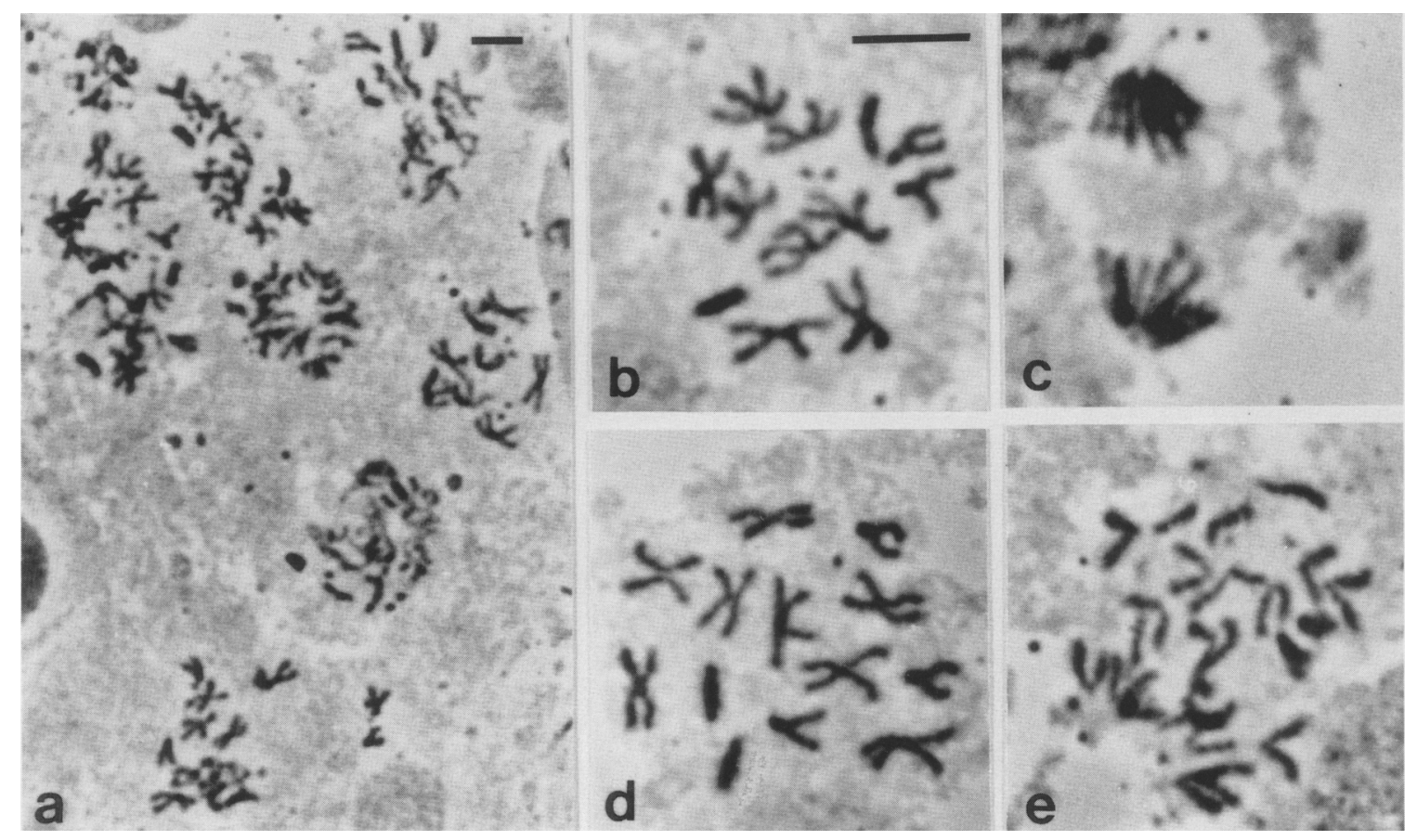

Figure 5. Mitotic figures in $1(1) d$ deg- 4 from noncolchicine, nonhypotonic-treated brains. $(a)$ High density of c-metaphases. $(b)$ Tetraploid metaphases. (c) Binsn/Y control anaphase. (d) Tetraploid-aneuploid metaphase (eight major autosomes, three $\mathrm{X}$, and two $\mathrm{Y}$ chromosomes). (e) Abnormal anaphase in 1(1)d deg-4. Bars, $5 \mu \mathrm{m}$. 
Figure 6. Mitotic figures from cells not treated with colchicine or hypotonic in $1(1) \mathrm{d} \mathrm{deg-10,1(1)d} \mathrm{deg-3}$ and not incubated with colchicine but treated with hypotonic in 1(3)13m-230. Diploid metaphase $(a)$ and polyploid/hyperploid metaphase $(b)$ in $1(1) \mathrm{d}$ deg-10; note the presence of chromosome breakage in $b$. Diploid metaphase $(c)$ and polyploid/hyperploid metaphase $(d)$ in $1(1) d$ deg-3. $(e, f)$ Diploid metaphases in $1(3) 13 m-230$; in $(f)$ one chromatid is broken into two parts of similar size, suggesting that breakage occurred at or near the centromere. Bar, $5 \mu \mathrm{m}$.

Another striking characteristic of these mutants is that polyploid anaphases are frequently multipolar (Table 2; Figs. 7 and 9|. Because the number of spindle poles in cells bearing these mutants appears at least to be correlated roughly with the level of polyploidy, it seems likely that spindle pole organizing centers and the genomes in these cells are being duplicated in parallel. In some preparations giant anaphases with more than 20 poles have been observed; one of these mitotic figures is shown in Figure 9. The accumulation of spindle pole organizing centers in highly polyploid cells in these mutants suggests that the duplication of spindle pole organizing centers is not dependant on the completion of chromosome segregation and cytokinesis. Moreover, the fact that polyploid cells organize multipolar spindles can explain part of the mutant phenotype: These cells are unlikely to succeed in cytokinesis, and so, once highly polyploid, chromosome number is only likely to increase with each division.

Finally, in several of the polyploid cells observed in

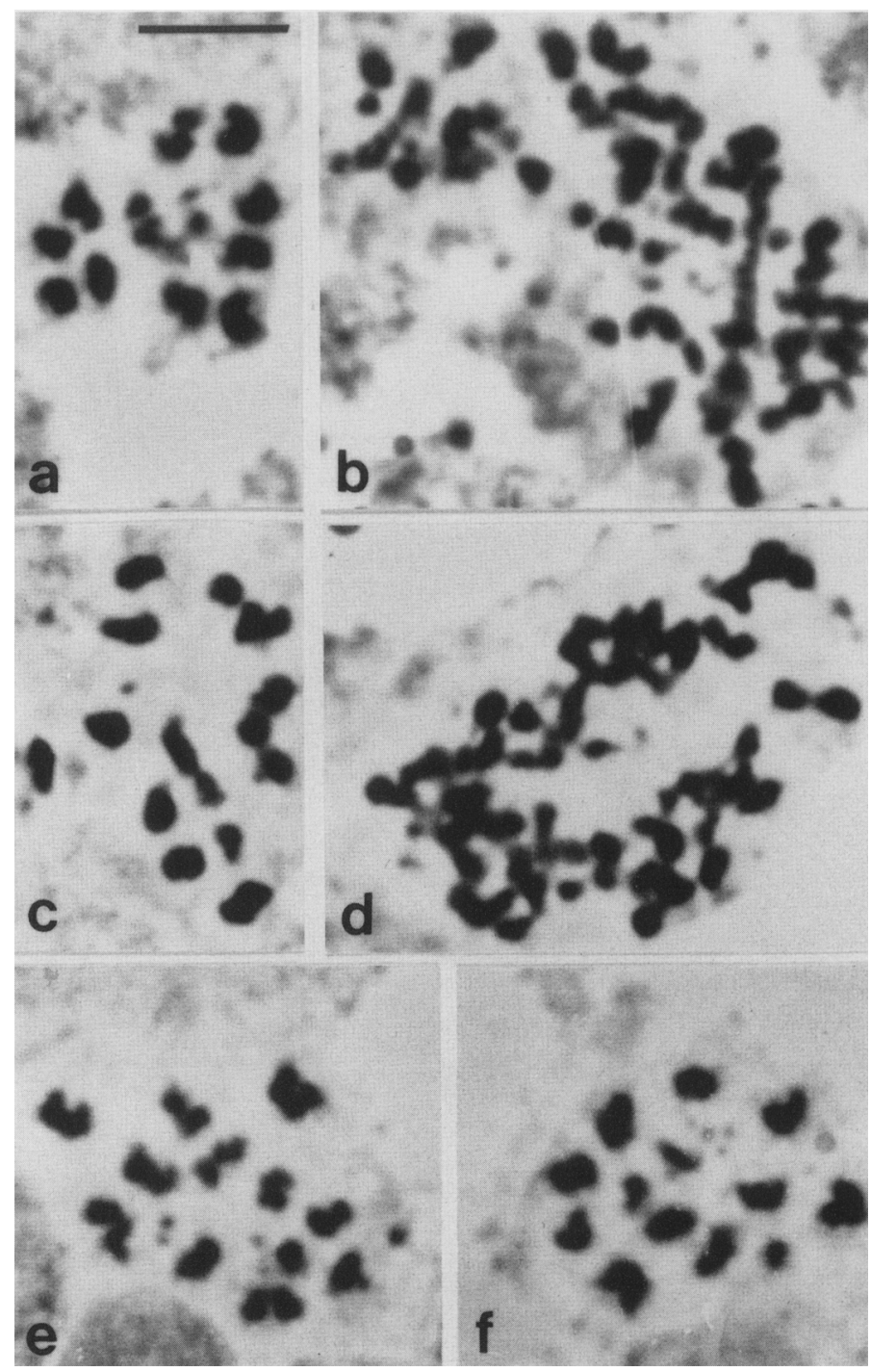

$1(3) 7 m-62$ there are broken chromosomes and/or acentric fragments, whereas in diploid cells of $1(3) 7 \mathrm{~m}-62$ the frequency of chromosome aberrations is not different from control $(\sim 0.5 \%)$. However, polyploid cells have more chromosomes than do diploid cells and so the difference in frequencies of breaks per cell in these two kinds of cells is inflated by the different sizes of their chromosome complements. Nevertheless, the absence of chromosome breakage in diploid cells suggests that chromosome breakage is occurring during the events that lead to polyploidization. Broken chromosomes and acentric fragments also have been observed in polyploid cells of $1(1) d$ deg-11, but the overall frequency of breaks detected in this mutant is lower than that found in $1(3) 7 m-62$.

Taken together, the available data suggest that these two mutants identify genes whose wild-type functions specify essential steps in chromosome segregation; one or both of these steps could be necessary for the completion of cytokinesis. 


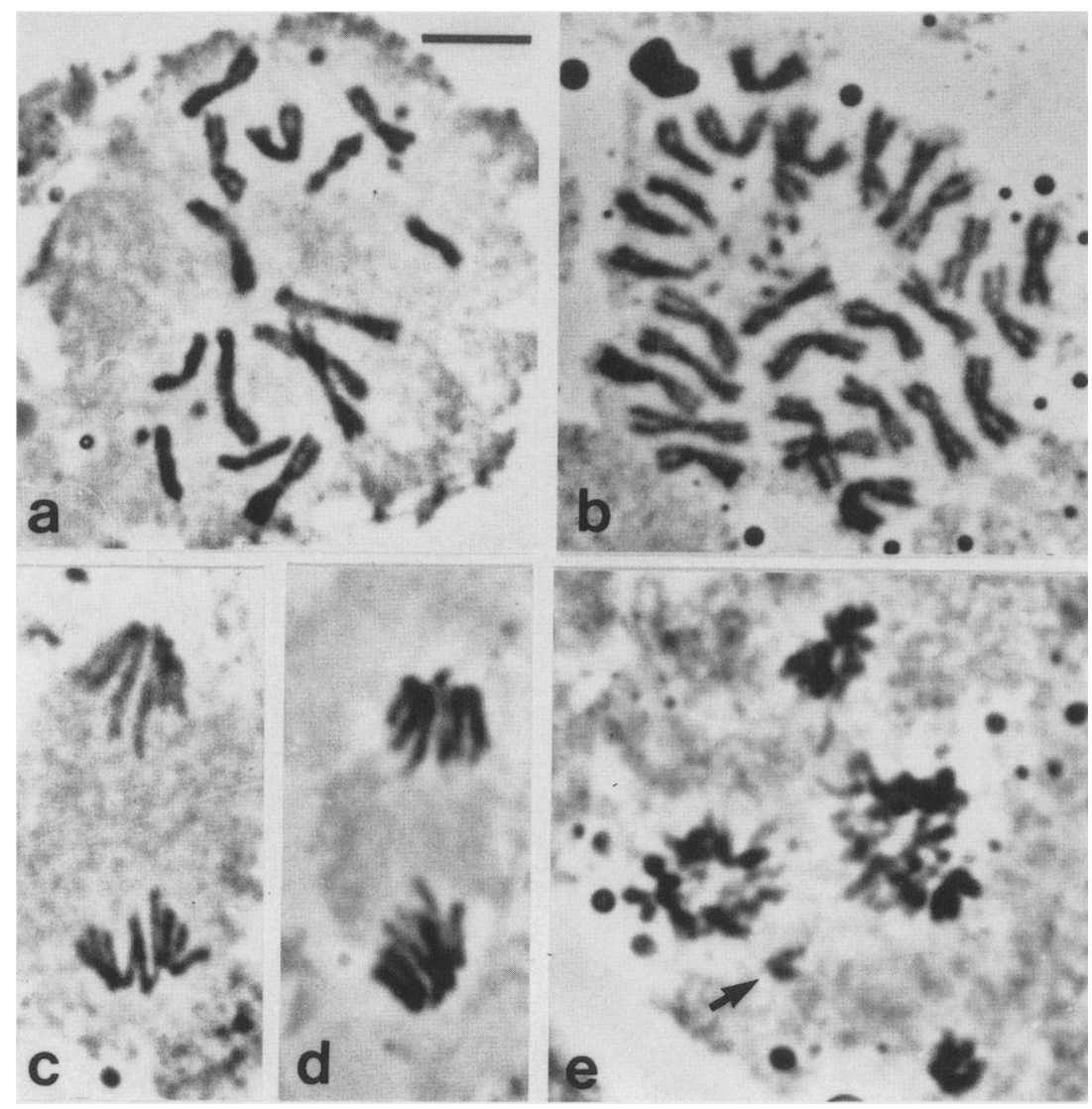

Figure 7. Mitotic figures in $1(3) 7 m-62$. (a) A noncolchicine nonhypotonic-treated hyperploid metaphase. $(b)$ A metaphase treated with hypotonic solution but not with colchicine. Note that the hypotonic treatment separates sister chromatids. $(c)$ Control anaphase. $(d, e)$ Anaphases in $1(3) 7 \mathrm{~m}-62$; (e) is a tetrapolar anaphase with a lagging chromosome (arrow). Bar, $5 \mu \mathrm{m}$.

\section{Discussion}

The operational definition employed here to identify mutants in essential mitotic cell-cycle functions differs from that routinely employed in fungal species and mammalian tissue cultures. In the latter systems, cellcycle mutants generally are defined as temperature-sensitive mutants that rapidly (within one to two cell cycles) arrest cell division at a unique point in the cell cycle following a shift to a restrictive temperature. Here we have also identified cell-cycle mutants by the fact that they cause an arrest or disruption of cell division. However, because the mutants examined are nonconditional lethals, the cells we screened probably have spent a substantial, but unknown, amount of time prior to our observing them in a situation in which an essential cellular function was absent. This raises the concern that some of the observed mitotic phenotypes are not due to the direct effects of cell-cycle mutants, but are more indirect consequences of mutations that alter some other aspect of cellular metabolism. Most generally, an observed phenotype could be the consequence of these cells being in an organism that may be sick and that certainly is going to die soon. However, for at least 12 of these mutants, their effects are sufficiently specific, on such central aspects of mitosis, and evident in such a substantial proportion of cells that we believe it is very likely these mutants identify loci with essential cellcycle functions. These include (1) those mutants showing severe defects in chromatin condensation and/ or high frequencies of chromosome breakage [1(1)d deg-1, mus-109ls, 1(3)e20, 1(3)k43, and 1(3)1902], (2) those mutants arresting cell division at metaphase $[1(1) d$ deg-3, 1(1)d deg-4, 1(1)d deg-10, and 1(3)13m-230], (3) those mutants producing highly polyploid cells [1(1)d deg-11 and $1(3) 7 m-62]$, and (4) the one mutant producing endoreduplicated cells [1(3)13m-281].

Although the mitotic effects of the remaining mutants [1(1)d discless-1, 1(1)C154, 1(3)IX-11 and the other mutants affecting chromatin condensation] are clear, it is less evident in all cases that they identify functions with direct roles in the chromosome cycle. At present the best evidence that at least some of these mutants [e.g., 1(1)d deg-9, 1(1)d deg-12, 1(1)het 2, 1(3)2004, 1(3)XII-10, and $1(3) 2612$ ] do identify real cell cycle functions is their quite specific chromosomal phenotypes.

Despite these ambiguities, our data establish that a substantial proportion of lethal mutations with a lethal phase during the late larval-early pupal period identify genes whose functions are important for mitotic cell division. With only one exception, [ $[1(3) \mathrm{m} 45]$, all of the mutants that affect cell division in the larval ganglia 


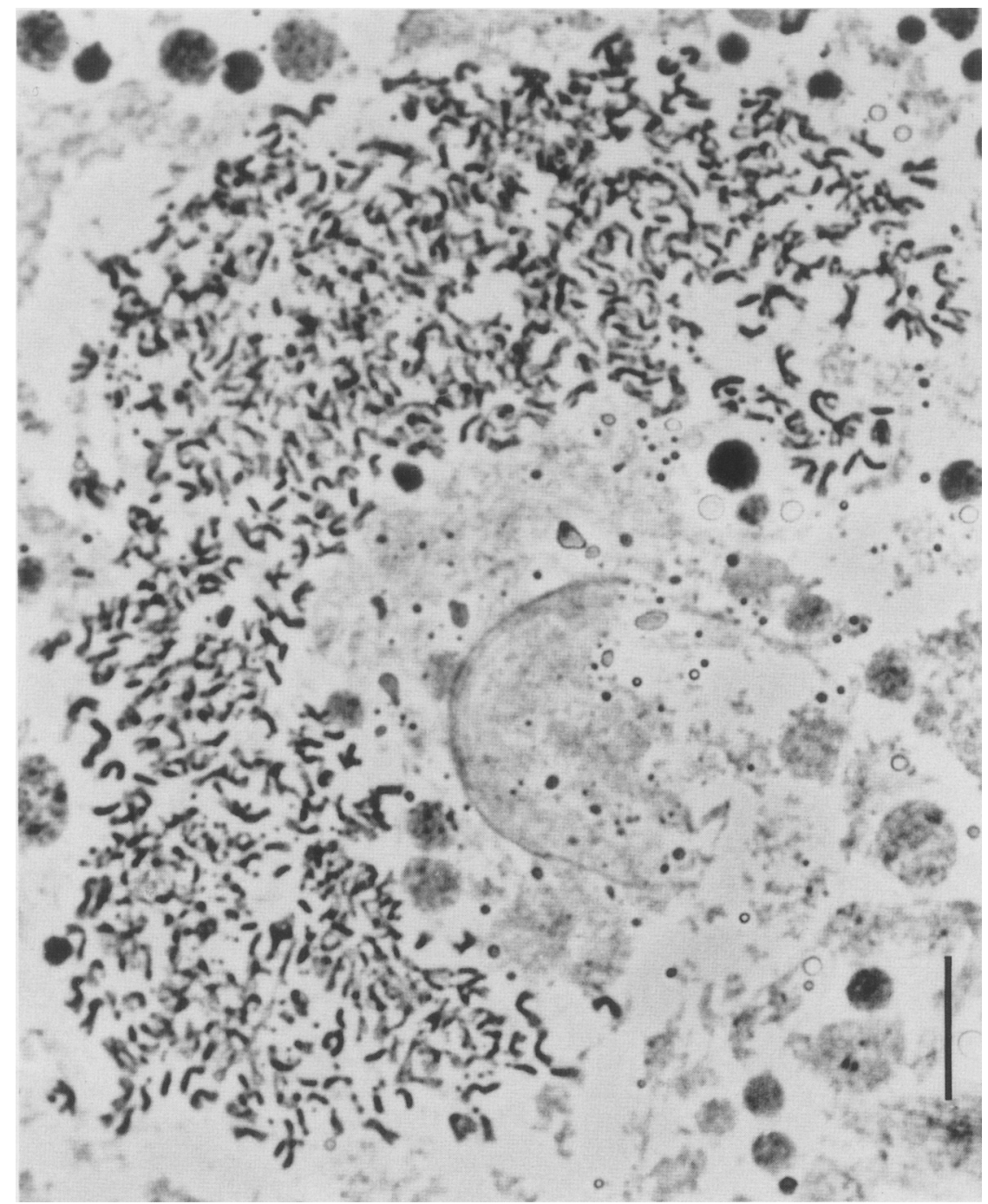

Figure 8. Giant metaphase of $1(3) 7 m-62$ containing $>1000$ chromosomes. This cell comes from a noncolchicine-treated brain incubated in hypotonic solution. Bar, $20 \mu \mathrm{m}$.

also exhibit imaginal discs that are reduced substantially in size. This suggests that these mutants may be affecting mitotic cell division in all the proliferating tissues of the larva. Moreover, that $68 \%$ (29 of 42) of all the mutants with missing, degenerate, or small imaginal discs affect mitosis suggests that the combined phenotype of late larval lethality and small or degenerate imaginal discs is almost diagnostic of a mutant in an essential cell-cycle function.

The finding that mutants affecting mitosis are frequent among late lethals is consistent with our hypothesis that the maternal genome has primary responsibility for the early embryonic cell divisions, including essentially all of the divisions needed to produce a larva, whereas it is primarily the functioning of the zygotic genome that directs the divisions of the imaginal disc and histoblast cells that are the progenitors of the adult (Baker et al. 1982). In at least one important respect, this dichotomy is an oversimplification of the situation: Larval cells undergo polytenization throughout larval life and thus mutants in genes concerned with bulk DNA synthesis should probably have lethal phases in the embryonic and/or early larval periods. Also, it should be emphasized that our success at finding mutants in essential cell-cycle functions among late lethals does not prove that the zygotic functioning of cell-cycle genes is dispensable for the formation of a viable larva. Such a proof would require showing that null mutants at cell-cycle genes also cause late larval lethality.

The present screen has provided 30 new mutants in genes that are necessary for normal mitotic cell division. Previous screens of meiotic mutants (Baker et al. 1978; Gatti 1979), mutagen-sensitive mutants (Baker and Smith 1979; Gatti 1979), temperature-sensitive lethals (Gatti et al. 1983; Smith et al. 1985), and nonconditional lethals (Ripoli et al. 1985; Gonzales et al. 1988; Sunkel and Glover 1988) for mutations affecting mitotic chromosome behavior identified genes whose wild-type functions were necessary for (1) the maintenance of mitotic chromosome integrity [i.e., mei-9, mei-41, 


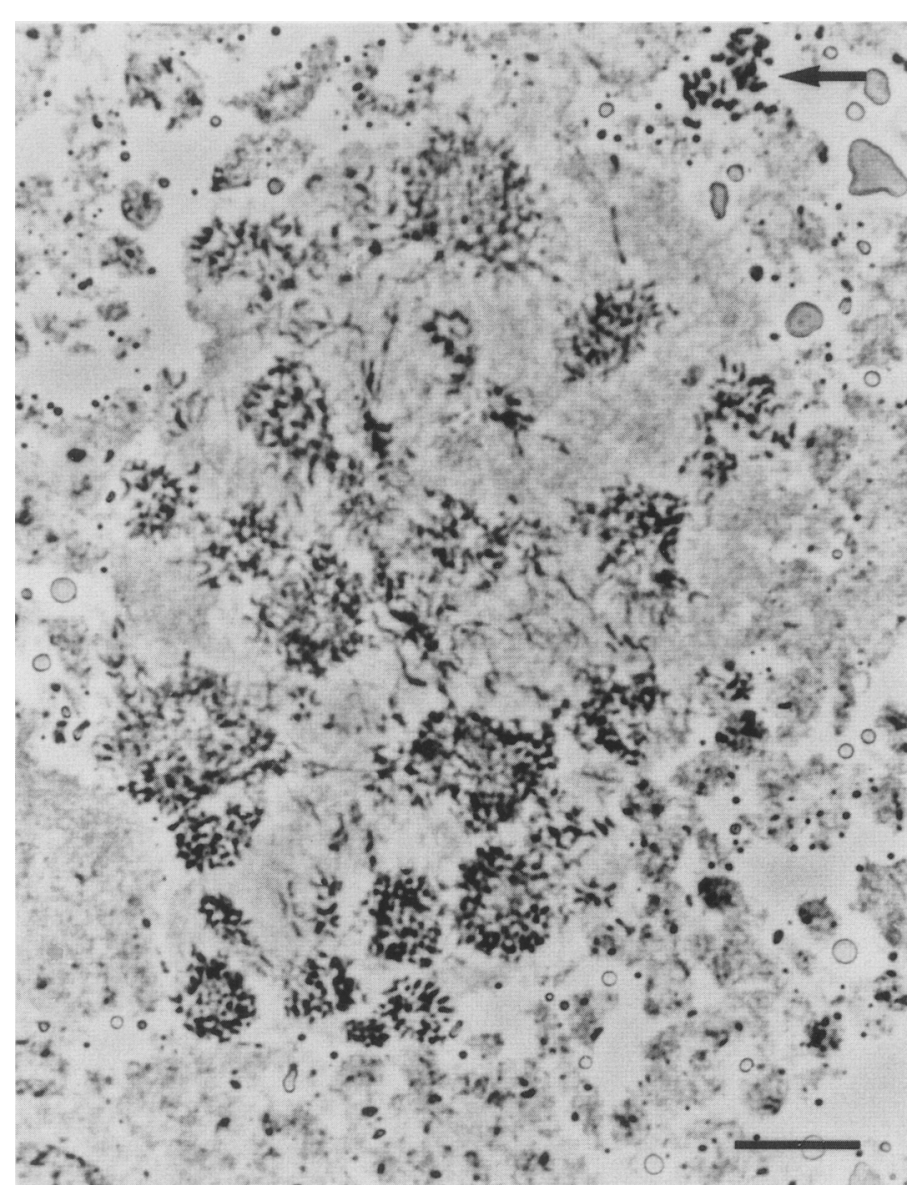

Figure 9. Giant multipolar anaphase with $>20$ spindle poles from $1(3) 7 m-62$. This arrow in the upper righthand corner points to a metaphase. Note that the metaphase chromosomes have two sister chromatids, whereas the anaphase chromosomes clearly are not duplicated. Bar, $20 \mu \mathrm{m}$. mus-102, mus-105, mus-109, and mit(1)14], (2) proper chromosome condensation [e.g., mit(1)4 and mus-101], (3) chromosome movement to the poles (asp, mgr, polo), and (4) the fidelity of chromosome segregation [1(1)zw10]. In addition to identifying a number of genes in these previously recognized categories of cell-cycle functions, our current screen has broadened the categories of identified genetic functions necessary for a normal chromosome cycle in $D$. melanogaster to include (1) genes necessary for progression through interphase, (2) a gene whose function is necessary for the coupling of chromosome duplication to the rest of the cell cycle $(1(3) 13 \mathrm{~m}-281)$, and (3) genes necessary for separation of chromosomes into daughter nuclei, but not chromosome duplication or movement [1(1)d deg-11 and $1(3) 7 m-62]$.

The phenotypes of the cell-cycle mutants now known in flies provide a set of information with which we can begin to put together a functional scheme of how the events of mitosis are controlled and integrated. There are two categories of mutants that need to be considered in putting together a flow chart of mitotic events. These are (1) mutants that arrest the cell cycle and (2) mutants that disrupt some aspect of the cell cycle but do not produce a cell-cycle arrest. For both types of mutants we have assumed that the mutant's phenotypes are caused by loss-of-function alleles and would not be qualitatively altered in more severe alleles (if the extant alleles are not null mutations). For mutations that block the occurrence of a particular step in the cell cycle, we can inquire whether or not other events of the cell cycle occur and can thus infer which aspects of the cell cycle are on dependent pathways and which are independently controlled (see, Hartwell 1978). For those mutants that do not cause cell-cycle blocks we infer that other cellcycle events are not on a dependent pathway with the execution of these gene's functions. In the following paragraphs we discuss the individual classes of cell-cycle mutants and the information that they provide about the control of the cell cycle.

Of the cell-division mutants identified in Drosophila, only a fraction of those found among late lethals leads to cytologically obvious simple cell-cycle blocks. These are the mutants that appear to arrest cells in interphase and those mutants producing metaphase blocks.

The mutants that arrest cells in interphase may fall into two qualitatively different categories with respect to the origins of their cell-cycle arrests. That is, this class of mutants, in addition to containing mutants producing interphase arrests by cell-cycle blocks equivalent to those found in other systems, may contain mutants that lead to cell-cycle arrests as a consequence of high frequencies of chromosome breakage. Our studies on mutants producing chromosome aberrations in Drosophila have shown that a chromosome break is a cell lethal event: Cells with broken metaphase chromo- 
somes only rarely produce daughter cells that reach the following metaphase (Gatti 1979; Baker et al. 1982). Thus, four of the mutations [1(3)e20, 1(3) K43, mus-109's, and 1(3)1902] identified here that exhibit frequent chromosome fragmentation, in addition to a low mitotic index, may be producing interphase arrests indirectly as a consequence of chromosome breakage. However, the mutant $1(3) I X-11$, which has a low mitotic index and no evident chromosome breakage, and the allelic mutants 1(1)discless and 1(1)C154, remain good candidates for mutations in cell-cycle genes that are necessary for the progression through interphase.

The mutation $1(3) 13 m-281$, in which the few mitotic figures observed were always endoreduplicated, is also likely to be in a gene necessary for progression through interphase. The phenotype of $1(3) 13 m-281$ is intriguing in that endoreduplication is potentially the origin of polyteny, which is observed in many cell types in Drosophila. Thus, the expression (or lack of expression) of the wild-type function of $1(3) 13 m-281$ may determine whether cells maintain a diploid mode of duplication or become polytenic. In this regard, it will be of substantial interest to determine whether the endoreduplication observed in the few mitotic figures seen in $1(3) 13 m-281$ neuroblasts is also present in the interphase cells of this mutant. In any case, the endoreduplication observed in $1 / 3) 13 m-281$ indicates that, in somatic cells of $D$. melanogaster, the initiation of DNA synthesis need not depend on the passage of cells through mitosis. This conclusion also has been reached from the analysis of the maternal effect mutation gnu, which leads to uncontrolled DNA synthesis but no nuclear division in embryos (Freeman et al. 1986; Freeman and Glover 1987).

The second class of mutations leading to cell-cycle blocks that have been identified in Drosophila are those producing metaphase arrest. In this category are four mutations reported here $[1(1) \mathrm{d}$ deg-4, $1(1) \mathrm{d}$ deg-3, $1(1) \mathrm{d}$ deg-10, and $1(3) 13 m-230$ ] and the mutant asp (Ripoll et al. 1985). These five mutations fall into three categories based on the properties of the metaphase arrests that they cause. In two mutants [1/1)d deg-4 and asp], chromosome morphology in arrested cells appears similar to that observed after a colchicine arrest. In addition, in both mutants a substantial proportion of cells have low levels of polyploidy, suggesting that arrested cells can revert to interphase and undergo an additional round(s) of DNA replication, as can cells that are blocked by colchicine. Two other mutants [1(1)d deg-3 and $1(1) d$ deg-10] share with asp and l(1) d deg-4 the property that arrested cells frequently exhibit low levels of polyploidy. However, $1(1) d$ deg-3 and 1(1)d deg-10 differ from asp and $1(1) \mathrm{d} \mathrm{deg-4}$ in that the chromosomes in arrested cells are much more condensed, sister chromatids are separated, and chromosome fragmentation is common and preferentially occurs at or near the centromere. The final mutation in this category, $1(3) 13 \mathrm{~m} 230$, is distinct from the other four mutations in this class in that no polyploid cells are observed. However, $1(3) 13 \mathrm{~m} 230$ shares with 1(1)d deg-3 and l(1)d deg-10 the property that the chromosomes in arrested cells are overcondensed, have separated sister chromatids, and chromosome breakage at or near the centromere is frequent. Unfortunately, $1(3) 13 m-230$ was lost before completion of cytological analysis and the available data do not permit us to infer the mitotic defect that leads to metaphase arrests but not to polyploidization.

In two mutants, $1(3) 7 m-62$ and $1(1) d$ deg-11, there appears to be occasional complete failure to segregate chromosomes to daughter cells, whereas the chromosome replication cycle and the duplication of spindle pole organizing centers proceed normally. This suggests that the events of the chromosome cycle are on a pathway independent of proper chromosome segregation. The maternal effect mutant gnu (Freeman et al. 1986), like $1(3) 7 \mathrm{~m} 62$ and $1(1) d$ deg-11, shows that both the chromosome cycle and the duplication of spindle pole organizing centers can proceed in the absence of nuclear division.

Another function whose execution does not appear to be necessary for continuation of the cell and chromosome cycles is identified by the mutations at the 1(1)zw10 locus (Smith et al. 1985). Mutant 1(1)zw10 alleles result in high frequencies of nondisjunction during mitotic cell division; this is thought to be due to the premature separation of sister chromatids and their subsequent independent assortment at anaphase.

The final major class of mutants that we have identified are those that affect chromosome condensation. These mutants generally exhibit mitotic indices lower than those in control but do not appear to arrest cell division. Whether this means that the cell cycle can proceed independently of chromosome condensation is unclear. On the one hand, to the degree that mutants affecting condensation render chromosomes more susceptible to breakage (many of them exhibit low levels of chromosome breakage, see Table 1), they will indirectly lead to cell-cycle arrests as described above. Thus, the reduced mitotic indices seen in many of these mutants may be only a secondary consequence of their effect on condensation. On the other hand our recognition of mutants affecting chromosome condensation is biased toward detecting either leaky mutants in early steps of condensation or mutants affecting late stages of this process; mutants that blocked the cell cycle by completely preventing an early step in condensation would appear at the light microscope level as if they were arresting cells in interphase.

The number and types of mutants available to date in Drosophila is sufficient to warrant a comparison with other systems in which cell-cycle mutants have been characterized. Although many of the structural molecules and enzymatic activities that are important for chromosome structure, and integral to the processes that comprise the cell cycle, show great evolutionary conservation, little is known about whether the control and integration of the events of the cell cycle is conserved similarly across species. As there are many ways one can a priori imagine that the executions of the events of the cell cycle could be integrated and coordinated, interspecific comparisons of the phenotypes pro- 
duced by cell-cycle mutations may be particularly informative as to whether these different organisms control the cell cycle in similar ways.

Cell-cycle mutants like those we have identified that arrest the cell cycle at metaphase are well represented among mammalian and yeast cell-cycle mutants (for mammalian examples, see Wang 1974; Abraham et al. 1983; Magnuson and Epstein 1984). Some of these mammalian mutants produce altered $\alpha$ - or $\beta$-tubulins and exhibit defective spindles (Abraham et al. 1983), whereas others do not exhibit any apparent spindle defect, but result in a clear metaphase arrest (i.e., the recessive lethal $O s$ in mice; Magnuson and Epstein 1984). None of the Drosophila mutants we have identified as producing a metaphase arrest maps near the site of a tubulin gene (Sanchez et al. 1980; Kalfayan and Wensink 1981). However, these mutants could be defective in any of the various other functions needed for spindle assembly and/or stability and thus have quite abnormal spindles. Although the presence of a poorly organized spindle is suggested by the distribution of chromosomes in the few anaphases we observed in 1(1) d deg-4, the proper assessment of the normality of the spindle in this mutant, as well as the other mutants producing metaphase arrests, must wait the direct examination of this organelle by electron microscopy or immunostaining. In fact, the biochemical characterization of spindle components in the Drosophila mutant asp (Ripoll et al. 1985), which has a mitotic phenotype similar to that of 1(1)d deg-4, has suggested that it alters a structural component of the spindle other than tubulins.

The mitotic phenotypes of many of the other Drosophila mutants described here also have striking similarities to those of mammalian cell-cycle mutants. For example, 1(3)13m-281, which produces endoreduplicated cells, is remarkably similar to E36ts41, a Chinese hamster cell-cycle mutant that goes through organized successive rounds of DNA replication but does not progress through the cell cycle when grown at restrictive temperature (Hirschberg and Marcus 1982). The induction of premature chromosome condensation in these cells revealed that they contain bundles of two or four chromosomes, i.e., appeared endoreduplicated.

Another category of Drosophila mutants having mammalian counterparts is that which affects the fidelity of chromosome segregation and produces highly polyploid cells. The two Drosophila mutants in this class, $1(3) 7 \mathrm{~m}-62$ and $1(1) \mathrm{d}$ deg-11, produce polyploid cells containing up to 500-1000 chromosomes. Examples of similar mammalian mutants are the Chinese hamster cell line mutants ts111 amd MS1-1, which produce large polynucleated or mononucleated cells and exhibit highly polyploid metaphases (Harzfeld and Buttin 1975; Thompson and Lindl 1976). Thus, these mutants appear to identify genes whose functions are necessary for cell division, but not the continuation of cycles of nuclear events. This appears to also be the case in yeast and other mammalian cell lines where mutants are known that produce repeated failures in chromosome segregation without arresting the cell cycle (for review, see Pringle and Hartwell 1971; Marcus et al. 1985).
Taken together the above comparisons indicate that there are substantial similarities between the terminal phenotypes exhibited by Drosophila and mammalian cell-cycle mutants. This is a striking observation because such terminal phenotypes are a reflection of how and whether the individual components in the complex arrays of events that comprise the cell cycle are dependent on a particular gene product. This suggests then that these organisms, despite their substantial biological differences, use a common genetic logic to regulate and integrate the events of the cell cycle.

\section{Methods}

Stocks

Flies were reared on a standard cornmeal, molasses, yeast, and proprionic acid medium at $25^{\circ} \mathrm{C}$. Except for the collections of late lethal mutations examined here and the third chromosome balancer TM6b (Craymer 1984), descriptions of all mutants and chromosomes may be found in Lindsley and Grell (1968).

Three collections of lethal mutants with lethal phases during the late larval-early pupal period were screened for mutants affecting mitosis. Most of the mutants screened were from the collection of third-chromosome mutants isolated by Shearn et al. (1971) and Shearn and Garen (1974). In addition, we screened those X-linked late lethals isolated by Kiss et al. (1976) that were reported to either reduce imaginal disc size or cause substantial cell death in imaginal discs. Also, we examined two X-linked mutants isolated by Stewart et al. (1972) [the three other surviving mutants from this collection had been already examined for their effects on mitosis (Baker et al. 1982)]. Finally, we examined a late lethal obtained from A. Schalet and two late lethals obtained from G. Lefevre (see Table 1).

In addition to the mutants whose characterization is reported in Table 1 , we attempted to screen 28 additional late lethals from these collections. However, no homozygous mutant larvae sufficiently mature for cytology were obtainable. Either these stocks have acquired additional lethals since their isolation or else the lethal larvae do not survive as well under our culture conditions.

All lethal mutants are kept in stable stocks by means of balancer chromosomes. For all X-linked mutants the balancer used was Binsn. Because the late lethal-bearing X chromosomes carry the marker white, which produces colorless Malpighian tubules, whereas Binsn does not, lethal-bearing male larvae were distinguishable from their nonlethal sibs by the color of their Malpighian tubules. Many of the chromosomes bearing third chromosome lethals carry the recessive Malpighian tubule color marker red, whereas the balancer chromosomes (either TM1 or TM3) in these stocks do not, so larvae homozygous for the lethal can be distinguished by Malpighian tubule color. Third chromosome lethals not marked with red were balanced over TM $6 b$ which carries the dominant larval, pupal, and adult shape marker Tubby (Tb), which allowed homozygous lethal (phenotypically non-Tubby) individuals to be distinguished from larvae heterozygous for the lethal (phenotypically Tubby).

\section{Cytology}

Metaphase chromosome figures were examined in aceto orcein squashes of colchicine-treated, third-instar larval ganglia prepared by conventional procedures (Gatti et al. 1974). To examine anaphases, ganglia were processed as for metaphase squashes with the exceptions that colchicine and hypotonic treatments were omitted. To control for treatment artifacts, 
control brains (balancer/Y males or mutant/balancer heterozygotes) and homozygous mutant brains of comparable sizes were treated together and squashed on the same slides.

To estimate the mitotic index in mutant brains relative to that in control brains, we counted all the mitotic figures present in comparable numbers of optic fields of control and mutant brains. An optic field is defined as the circular area seen under a phase contrast Neofluar $100 \times$ oil-immersion Zeiss objective, using $10 \times$ oculars and the Optovar set at $1.25 \times$. The mitotic indices reported in Table 1 are relative mitotic indices and represent the ratio of the mitotic index in a mutant to the mitotic index of its control.

Because in some mutants mitotic figures were rare, or even absent, the number of mitotic figures examined per mutant for the normality of chromosome behavior varied. In all the mutants for which the frequency of mitotic figures was between 0.5 and 1.5 times that seen in its control, at least 300 metaphases were examined for the normality of chromosome number, integrity, and condensation.

\section{Acknowledgments}

We are grateful to A. Shearn, I. Kiss, A. Schalet, and G. Lefevre for providing us with the late lethals examined in this paper, and to A.T.C. Carpenter for her thoughtful comments on the manuscript. Part of the work reported in this paper was undertaken during the tenure of an American Cancer SocietyEleanor Roosevelt International Cancer Fellowship awarded by the International Union Against Cancer; this work was also supported by a grant from National Institutes of Health. We would like to acknowledge the involvement of D. A. Smith in the initial phase of this work.

\section{References}

Abraham, I., M. Marcus, F. Cabral, and M.M. Gottesman. 1983. Mutations in $\alpha$ - and $\beta$-tubulin affect spindle formation in Chinese hamster ovary cells. J. Cell Biol. 97: 1055-1061.

Baker, B.S., A.T.C. Carpenter, and P. Ripoli. 1978. The utilization during mitotic cell division of loci controlling meiotic recombination and disjunction in Drosophila melanogaster. Genetics 90: 531-578.

Baker, B.S. and D.A. Smith. 1979. The effects of mutagen sensitive mutants of Drosophila melanogaster in nonmutagenized cells. Genetics 92: 833-847.

Baker, B.S., D.A. Smith, and M. Gatti. 1982. Region-specific effects on chromosome integrity of mutations at essential loci in Drosophila melanogaster. Proc. Natl. Acad. Sci. 79: $1205-1209$.

Brinkley, B.R., S.M. Cox, and D.A. Pepper. 1980. Structure of the mitotic apparatus and chromosomes after hypotonic treatment of mammalian cells in vitro. Cytogenet. Cell Genet. 26: 165-176.

Craymer, L. 1984. Third multiple Six, b structure. Drosophila Inform. Serv. 60: 234.

Fantes, P. 1984. cell-cycle control in Schizosaccharomyces pombe. In The microbial cell cycle, (ed. P. Nurse and E. Streiblova), pp. 109-125. CRC Press, Boca Raton, Florida.

Freeman, M., C. Nusslein-Volhard, and D.M. Glover. 1985. The dissociation of nuclear and centrosomal division in gnu, a mutation-causing giant nuclei in Drosophila. Cell 46: 457468.

Freeman, M. and D. Glover. 1987. The gnu mutation of Drosophila causes inappropriate DNA synthesis in unfertilized and fertilized eggs. Genes Dev. 1: 924-930.

Gatti, M., C. Tanzarella, and G. Olivieri. 1974. Analysis of the chromosome aberrations induces by X-rays in somatic cells of Drosophila melanogaster. Genetics 77: 701-719.
Gatti, M. 1979. Genetic control of chromosome breakage and rejoining in Drosophila melanogaster. I. Spontaneous chromosome aberrations in X-linked mutants defective in DNA metabolism. Proc. Natl. Acad. Sci. 76: 1377-1381.

Gatti, M., S. Pimpinelli, and B.S. Baker. 1980. Relationships among chromatid interchanges, sister chromatid exchanges, and meiotic recombination in Drosophila melanogaster. Proc. Natl. Acad. Sci. 77: 1575-1579.

Gatti, M., D.A. Smith, and B.S. Baker. 1983. A gene controlling condensation of heterchromatin in Drosophila melanogaster. Science 221: 83-85.

Gonzalez, C., J. Casal, and P. Ripoli. 1988. Functional monopolar spindles caused by mutation in $m g r$, a cell division gene of Drosophila melanogaster. I. Cell Sci. 89: 34-47.

Hartwell, L.H. 1974. Saccharomyces cerevisiae cell cycle. Bacteriol. Rev. 38: 164-198.

. 1978. Cell division from a genetic perspective. I. Cell Biol. 77: 627-637.

Harzfeld, J. and G. Buttin. 1975. Temperature-sensitive cellcycle mutants: a Chinese hamster cell line with a reversible block in cytokinesis. Cell 5: 123-129.

Hirschberg, J. and M. Marcus. 1982. Isolation by a replicaplating technique of Chinese hamster temperature-sensitive cell-cycle mutants. J. Cell Physiol. 113: 159-166.

Kalfayan, L. and P.C. Wensink. 1981. $\beta$-tubulin genes of Drosophila. Cell 24: 97-106.

Kiss, I., G. Bencze, E. Fekete, A. Fodor, J. Gausz, P. Maroy, J. Szabad, and J. Szidonya. 1976. Isolation and characterization of X-linked lethal mutants affecting differentiation of the imaginal discs in Drosophila melanogaster. Theor. Appl. Genet. 48: 217-226.

Kiss, I., J. Szabad, and J. Major. 1978. Genetic and developmental analysis of puparium formation in Drosophila. Molec. Gen. Genet. 164: 77-83.

Lindsley, D.L. and E.H. Grell. 1968. Genetic variations in Drosophila melanogaster. Carnegie Inst. Wash. Publ. 627.

Lindsley, D. and G. Zimm. 1986. The genome of Drosophila melanogaster. Part 2: lethals; maps. Drosophila Inform. Serv. 64.

Ling, V. 1981. Mutants as an investigative tool in mammalian cells. In Mitosis/cytokinesis. (ed. A.M. Zimmerman and A. Forer\}, pp. 197-209. Academic Press, New York.

Magnuson, T. and C.J. Epstein. 1984. Oligosyndactyly: a lethal mutation in the mouse that results in mitotic arrest very early in development. Cell 38: 823-833.

Marcus, M., A. Fainsod, and G. Diamond. 1985. The genetic analysis of mammalian cell-cycle mutants. Annu. Rev. Genet. 19: 389-421.

Mazia, D. 1961. Mitosis and the physiology of cell division. In The cell, (ed. J. Brachet and A.E. Mirsky), vol. 3, pp. 77-612. Academic Press, New York.

Moir, D. and D. Botstein. 1982. Determination of the order of gene function in the yeast nuclear division pathway using $c s$ and ts mutants. Genetics 100: 565-577.

Moir, D., S.E. Stewart, B.C. Osmond, and D. Botstein. 1982. Cold-sensitive cell-division cycle mutants of yeast: isolation, properties and pseudoreversion studies. Genetics 100: $547-563$

Nagl, W. 1978. Endopolyploidy and polyteny in differentiation and evolution. North Holland, Amsterdam.

Nurse, P. 1985. cell-cycle control in yeast. Trends Genet. 1: 51-55.

Oakley, B.R. 1981. Mitotic mutants. In Mitosis/cytokinesis. (ed. A.M. Zimmerman and A. Forer), pp. 181-196. Academic Press, New York.

Pickett-Heaps, J.D., D.H. Tippet, and K.R. Porter. 1982. Rethinking mitosis. Cell 29: 729-744. 
Pringle, J.R. and L.H. Hartwell. 1981. The Saccharomyces cerevisiae cell cycle. In Molecular biology of the yeast Saccharomyces. Life cycle and inheritance. (ed. J.N. Strathern, E.W. Jones, and J.R. Broach), pp. 97-142. Cold Spring Harbor Laboratory, Cold Spring Harbor, New York.

Ripoll, P., S. Pimpinelli, M.M. Valdivia, and J. Avila. 1985. A cell division mutant of Drosophila with a functionally abnormal spindle. Cell 41: 907-912.

Sanchez, L., J.E. Natzle, D.W. Cleveland, M.W. Kirschner, and B.J. McCarthy. 1980. A dispersed multigene family encoding tubulin in Drosophila melanogaster. Cell 22: 845-854.

Schwarzacher, H.G. and W. Schnedl. 1965. Endoreduplication in human fibroblast cultures. Cytogenetics 4: 1-8.

Shearn, A. and A. Garen. 1974. Genetic control of imaginal disc development in Drosophila. Proc. Natl. Acad. Sci. 71: $1393-1397$.

Shearn, A., T. Rice, A. Garen, and W. Gehring. 1971. Imaginal disc abnormalities in lethal mutants of Drosophila. Proc. Natl. Acad. Sci. 68: 2594-2598.

Simchen, G. 1978. cell-cycle mutants. Annu. Rev. Genet. 12: $161-191$.

Smith, D.A., B.S. Baker, and M. Gatti. 1985. Mutations in genes controlling essential mitotic functions in Drosophila melanogaster. Genetics 110: 647-670.

Stewart, M., C. Murphy, and J.W. Fristrom. 1972. The recovery and preliminary characterization of $\mathrm{X}$ chromosome mutants affecting imaginal discs of Drosophila melanogaster. Dev. Biol. 27: 71-83.

Sunkel, C. and D.M. Glover. 1988. polo, a mitotic mutant of Drosophila displaying abnormal spindle poles. I. Cell Sci. 89: 25-38.

Szabad, J. and P.J. Bryant. 1982. The mode of action of 'discless' mutations in Drosophila melanogaster. Dev. Biol. 93: 240256.

Thompson, L.H. and P.A. Lindl. 1976. A CHO cell mutant with a defect in Cytokinesis. Somatic Cell Genet. 2: 387-400.

Wang, R.J. 1974. Temperature-sensitive mammalian cell line blocked in mitosis. Nature 248: 76-78.

Wissinger, W.L. and R.J. Wang. 1983. cell-cycle mutants. Int. Rev. Cytol. Suppl. 15: 91-113. 


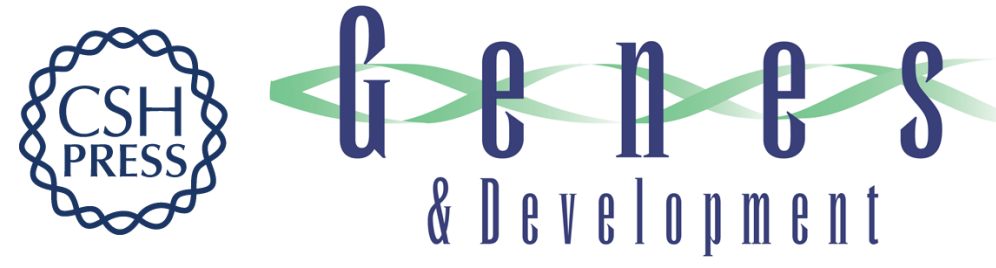

\section{Genes controlling essential cell-cycle functions in Drosophila melanogaster.}

M Gatti and B S Baker

Genes Dev. 1989, 3:

Access the most recent version at doi:10.1101/gad.3.4.438

References This article cites 38 articles, 17 of which can be accessed free at:

http://genesdev.cshlp.org/content/3/4/438.full.html\#ref-list-1

License

Email Alerting

Service

Receive free email alerts when new articles cite this article - sign up in the box at the top right corner of the article or click here.

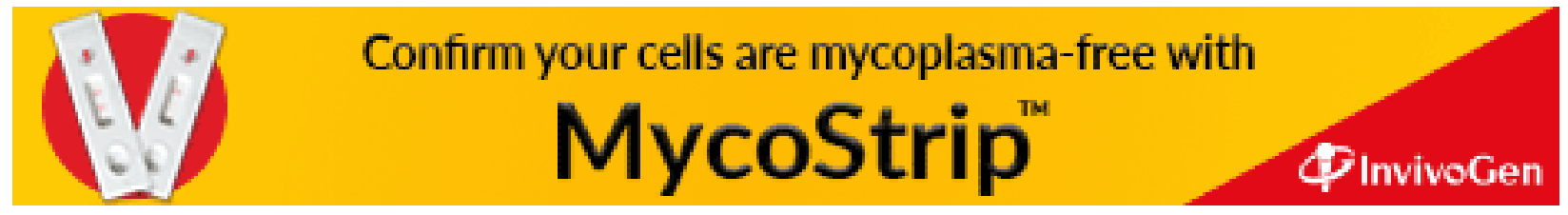

